\title{
Automatic Detection of Exudates from Digital Color Fundus Images
}

\author{
Ahmed S. El Sisy \\ Department of Biomedical \\ Engineering \\ Faculty of Engineering \\ Helwan University, Cairo, \\ Egypt
}

\author{
Nancy M. Salem \\ Department of Biomedical \\ Engineering \\ Faculty of Engineering \\ Helwan University, Cairo, \\ Egypt
}

\author{
Ahmed F. Seddik \\ Department of Biomedical \\ Engineering, \\ Faculty of Engineering \\ Helwan University, Cairo, \\ Egypt
}

\begin{abstract}
Diabetic retinopathy is a widespread disease that may cause blindness. Early diagnosis and treatment will reduce its side effects and protect the eye. In this paper, a new algorithm for exudates detection is proposed. In the preprocessing step, the green channel of the color image is used, and then median filter followed by Contrast Limited Adaptive Histogram Equalization (CLAHE) is applied. The K-means clustering technique is used to select exudates objects. Optic disc is localized using maximum entropy filter and morphological closing. It is demonstrated that combining the K-means with CLAHE of the median filtered image results in $99.39 \%$ correct exudates. Experimental results show a reliable and accurate method for segmenting exudates from color retinal images. Performance of the proposed method is evaluated using a set of 52 images from a publicly available dataset STARE.
\end{abstract}

\section{Keywords}

Diabetic Retinopathy, Exudates detection, Entropy filter, Kmeans clustering

\section{INTRODUCTION}

Digital image processing techniques play an important role in the medical area. In ophthalmology, it is used in screening of glaucoma [1], Macular Edema [2], and Diabetic Retinopathy (DR) [3]. DR is a disease caused by effect of high blood glucose level in diabetic patient on retinal blood vessels, that passes through many stages and can lead finally to blindness [4]. One of the main early features of the DR is exudates [5]. The damaged blood vessels of the retina leak proteins and lipids which deposit forming yellow white spots with relatively well defined margins that is defined as exudates [5].

Early diagnosis and treatment of DR will reduce the risk of blindness [6]. Periodic screening helps in early detection and the automatic process in screening programs is a costeffective method that can also save time [7]. In the literature, many techniques have been proposed for exudates detection.

Sopharak et al. [8] proposed a method for the detection of diabetic retinopathy by detecting the presence of exudates in the retinal image. The preprocessing consists of conversion of RGB to gray scale and median filtering is carried out to remove noise. Histogram equalization is the next step. Exudates and optic disc had the highest intensity values in the preprocessed image. The optic disc (OD) was eliminated based on the texture, as the optic disc has a smooth texture. Based on entropy the texture was analyzed, low entropy is an indication that the region is smooth. Otsu's binarization algorithm was used to remove complex regions in the image. The binarized image has many components of which the largest connected component was selected and circularity was found.

Karegowda et al. [9] converted RGB to HSI then applied salt and pepper noise to the I band followed by median filter. Then applied contrast limited adaptive histogram equalization CLAHE, the optic disc was extracted using a matlab function, pixel features were extracted, and then classification using artificial neural network is performed.

Eadgahi et al. [10] used two phases. In the first phase morphological operations on the green channel were used to eliminate vessels, bright and yellow objects with higher intensities were extracted. In the second phase, the optic disc was eliminated using blood vessel structure and entropy. In the work proposed in [6], the red channel is used for OD detection then the green channel is used for exudates detection. Median filtering was applied to reduce noise on the green component. OD has been eliminated then exudates have been extracted.

Tripathi et al. [11] used the green component of image then Gaussian smoothing filter followed by contrast enhancement to reduce noise and uneven illumination. Candidate exudates were detected by using Differential Morphological Profile (DMP). El Abbadi et al. [12] used the green channel because it had the best contrast. Binary image was obtained using threshold value equal 110. Texture features were used to differentiate between true and false exudates. Histogram equalization and the $k$-means clustering were used in the work proposed in [13]. Then a neural network is used for exudates detection.

Franklin et al. [14] converted from the RGB to Lab color space. The luminosity layer is replaced with the processed data, and then converts the image back to the RGB color space. Contrast enhancement has been done using CLAHE. Features like color, size, shape, edge strength and texture were used with three layers feed forward neural network to classify candidate regions into exudates and non exudates. The method is evaluated using DIARETDB1 database, a mean sensitivity of $96.3 \%$, mean specificity of $99.8 \%$ and predictive value of $93.7 \%$ have been achieved.

Zhang et al. [15] detected exudates through three stages; preprocessing, exudates candidates detection, and finally classification. Removing of all dark structures and bright artifacts were done in the preprocessing stage, morphological top hat was used to detect small exudates candidates from 
green channel of fundus image. Large exudates candidates were extracted by applying mean filter followed by reconstruction. Classification was done using intensity features and geometric features followed by machine learning technique. The method is evaluated using DIARETDB1_V2, MESSIDOR and HEI-MED, with corresponding area under curve (AUC) of 0.95, 0.93 and 0.94 respectively.

In this paper, a new method is proposed to detect exudates as a symptom of diabetic retinopathy from color fundus images. This paper is organized as follows; Section 2 describes the proposed method. Section 3 presents experimental results and conclusions are given in Section 4.

\section{PROPOSED METHOD}

In order to identify exudates from color fundus image, a reliable segmentation method is required. Segmentation is the procedure of partitioning an image into a set of regions. Figure 1 shows an original normal image and abnormal image. Figure 2 shows the block diagram of proposed method.

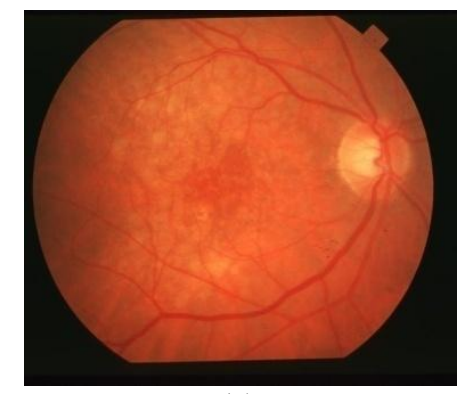

(a)

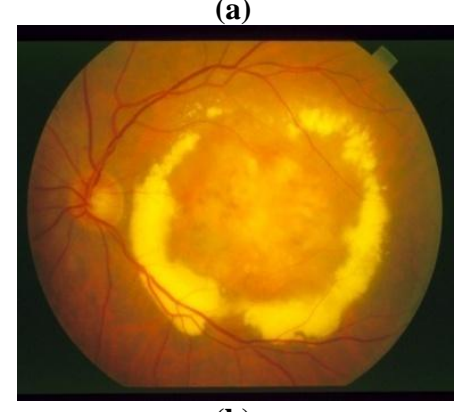

(b)

Fig 1: (a) Normal image, and (b) Abnormal image

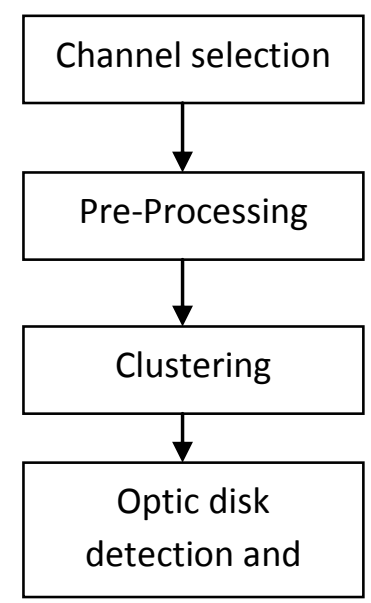

Fig 2: Block diagram of the proposed method

\subsection{Pre-processing step}

Fundus images often show luminosity variation, differences in contrast and noise. Pre-processing is a mandatory step to overcome these problems. The median filter is a type of filters that is used to remove noise [16] and it is calculated as:

$$
\widehat{\mathcal{F}}(x, y)=\operatorname{median}_{(s, t) \in s_{x y}}\{g(s, t)\}
$$

where $\hat{\mathcal{F}}(\mathrm{x}, \mathrm{y})$ is the filter response at a given coordinate, Sxy is an $m \times n$ sub-image of the input image g. $x$ and $y$ are the coordinate in the input image.

Contrast limited adaptive histogram equalization (CLAHE) is used for improving the contrast of an image. It is commonly used in ophthalmology [17]. CLAHE technique partitions the images into tiles and applies the histogram equalization to each one. It calculates many histograms, each corresponds to an individual tile of the retinal image and then applies them to redistribute the intensity value of the image [14]. So, it becomes significant to enhance the local contrast of the image and thereby gives detailed information of the exudates presented in the fundus image.

\subsection{Clustering}

Clustering is partitioning a group of data [5]. The K-means clustering divides the input data into many groups depending on the squared Euclidean distance [5]. K-means clustering algorithm [18] is working as follows:

Let $X=\left\{x_{1}, x_{2}, x_{3}, \ldots, x_{n}\right\}$ be the set of data points and $V=$ $\left\{\mathrm{v}_{1}, \mathrm{v}_{2}, \ldots, \mathrm{v}_{\mathrm{c}}\right\}$ be the set of centers.

1. Arbitrarily select 'c' cluster centers.

2. Calculate the distance between each data point and cluster centers.

3. Specify the data point to the cluster center whose distance from the cluster center is minimal of all the cluster centers.

4. Calculate again the new cluster center using:

$$
\mathrm{v}_{\mathrm{i}}=\left(1 / \mathrm{p}_{\mathrm{i}}\right) \sum_{\mathrm{j}=1}^{\mathrm{p}_{\mathrm{i}}} \mathrm{x}_{\mathrm{i}}
$$

where, 'pi' represents the number of data points in $i^{\text {th }}$ cluster.

5. Calculate again the distance between each data point and new cluster centers.

6. If no data point was reassigned then stop, otherwise repeat from step 3.

\subsection{Optic disk detection}

The Optic disc localization is an important step in automatic extraction of retinal exudates [19]. In this paper, the maximum entropy filter [20] is used for localization of the OD. Entropy can be calculated using Eqn. 3 where $P_{i}$ is the probability that the difference between two adjacent pixels is equal to $i$.

$$
\text { Entropy }=-\sum_{\mathrm{i}} \mathrm{P}_{\mathrm{i}} \log _{2} \mathrm{P}_{\mathrm{i}}
$$

The maximum entropy filter was applied on the green channel of the input image followed by removing small object with area less than 30 pixels. Then morphological closing is applied on the output image. Figure 3 shows the steps of the OD detection. Figure (3-a) shows the output image after applying the maximum entropy filter, and Fig. (3-b) shows OD localization by selecting object with maximum circularity and then circle fitting. 

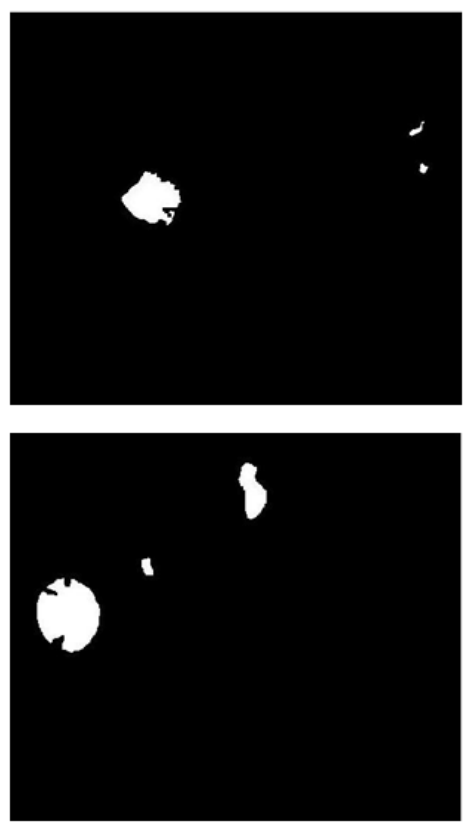

(a)
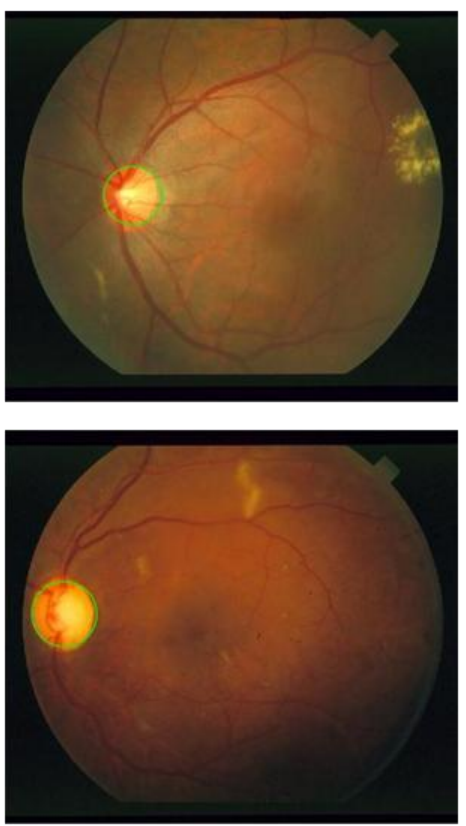

(b)

Fig 3: OD detection (a) Binary image, and (b) Circle fitting

\section{EXPERIMENTAL RESULTS}

A set of 52 images from a publicly available database is used. The STARE [21] database consists of 81 fundus images (31 normal and 50 diseased), The images were captured by a Topcon TRV-50 fundus camera at $35^{\circ}$ field of view. Each image was digitized to produce a $605 \times 700$ pixel image. Median filter is applied to the green channel, followed by contrast limited adaptive histogram equalization. The median filter with size $5 \times 5$ is used. Figure 4 shows the pre-processing step.

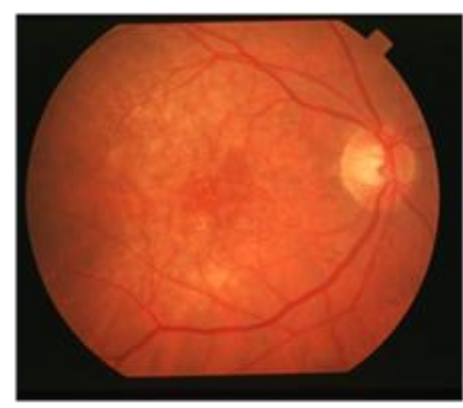

(a)

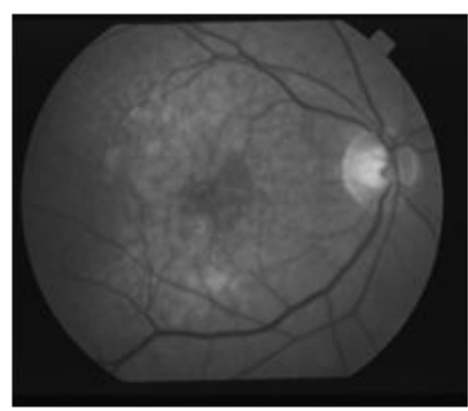

(c)
The median filter results in smoothing and noise reduction. The output image after contrast limited adaptive histogram equalization shows contrast improvement. After the preprocessing, a clustering technique is used. In this study, the number of clusters in the image equal 5 . Figure 5 shows the result after the K-means clustering. The output from the $\mathrm{K}$ means clustering shows different clusters that represent many regions of interest in the test image. The cluster that represents the exudates is selected and shown in Fig. (5-b).

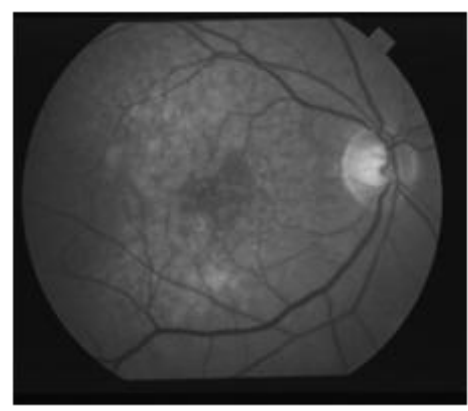

(b)

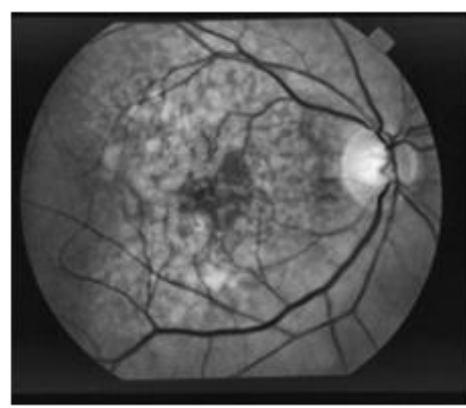

(d)

Fig 4: Preprocessing step: (a) RGB image, (b) Green channel image, (c) Median filter image, and (d) CLAHE image 

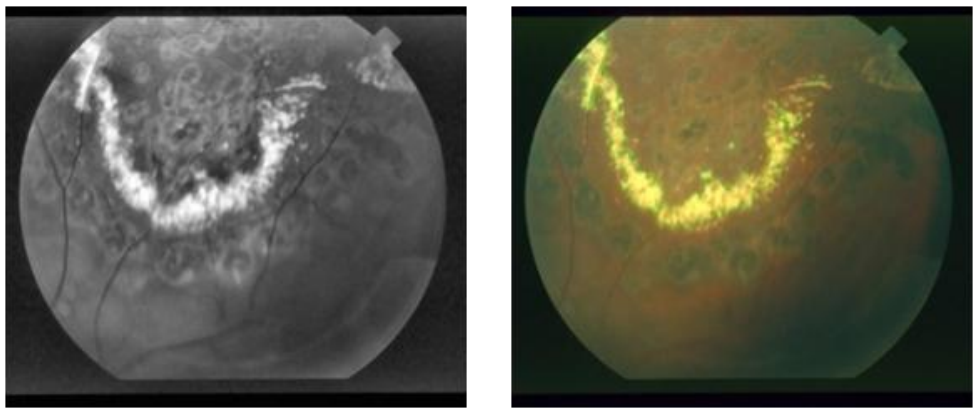

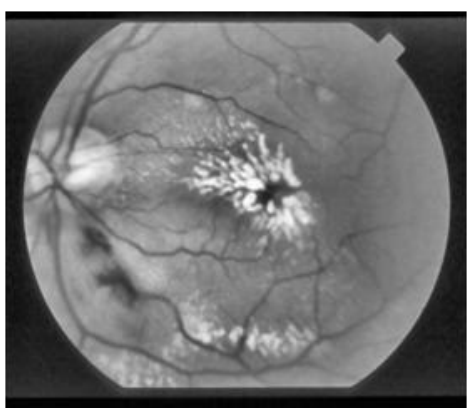

(a)

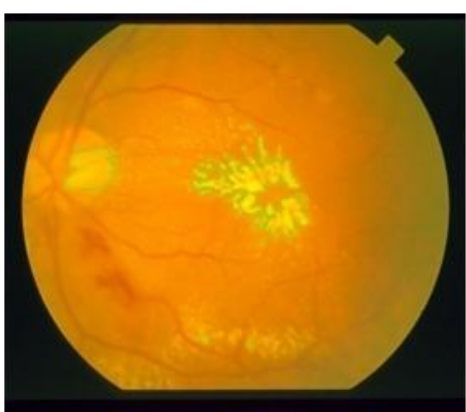

(b)

Fig 5: (a) CLAHE image, and (b) Exudates detection using K-means clustering

For performance evaluation of the proposed method, the total number of exudates pixels is calculated. Objects that belong to exudates, background, or optic disc were manually labeled. Results are summarized in Table 1. Other methods in the literature have been implemented and results are tabulated in Table 2.

Table 1. Results of the proposed method

\begin{tabular}{|c|c|c|}
\hline Type & No. of pixels & Percentage \\
\hline Exudates & 590055 & $88.7 \%$ \\
\hline Non-exudates & 4028 & $0.605 \%$ \\
\hline Optic disk & 71142 & $10.69 \%$ \\
\hline Total no. of pixels & 665225 & $100 \%$ \\
\hline
\end{tabular}

Table 2. Results of other methods

\begin{tabular}{|l|c|c|c|c|}
\hline \multirow{2}{*}{ Method } & \multicolumn{4}{|c|}{ No. of pixels } \\
\cline { 2 - 5 } & Exudates & $\begin{array}{c}\text { Non- } \\
\text { exudat }\end{array}$ & OD & Total \\
\hline $\begin{array}{l}\text { Amel et al. } \\
\text { [22] }\end{array}$ & 245361 & 55139 & 38271 & 338771 \\
\hline $\begin{array}{l}\text { Vimala and } \\
\text { Mohideen [5] }\end{array}$ & 211700 & 34504 & 35782 & 281986 \\
\hline $\begin{array}{l}\text { Ramasubraman } \\
\text { ian and } \\
\text { Mahendran [7] }\end{array}$ & 242346 & 47528 & 35023 & 324897 \\
\hline
\end{tabular}

ivin uic pic vivus tavics, it is ucmunisuaicu uat combining the K-means clustering with the CLAHE of the median filtered image results in $93.66 \%$ correct exudates. After removing the OD; results improved to be $99.39 \%$ correct exudates.

\section{CONCLUSIONS}

In this paper, a segmentation method for exudates detection is proposed. There steps were applied, pre-processing and clustering. The green channel is used in the pre-processing followed by median filter and contrast limited adaptive histogram equalization. In the clustering step, the K-means clustering was used. The optic disk is localized by using the maximum entropy filter.

\section{ACKNOWLEDGMENTS}

The authors would like to thank A. Hoover for making the STARE database publicly available.

\section{REFERENCES}

[1] Haleem M. S., Han L., van Hemert J., and Li B., "Automatic extraction of retinal features from colour retinal images for glaucoma diagnosis: a review," Computerized Medical Imaging and Graphics, vol. 37, pp. 581-596, 2013.

[2] Deepak K. S. and Sivaswamy J., "Automatic assessment of macular edema from color retinal images," Medical Imaging, IEEE Transactions on, vol. 31, pp. 766-776, 2012.

[3] Ahmad A., Mansoor A. B., Mumtaz R., Khan M., and Mirza S., "Image processing and classification in diabetic retinopathy: a review," in Visual Information Processing (EUVIP), 2014 5th European Workshop on, 2014, pp. 16.

[4] Tariq A., Akram M. U., and Javed M. Y., "Computer aided diagnostic system for grading of diabetic retinopathy," in Computational Intelligence in Medical Imaging (CIMI), 2013 IEEE Fourth International Workshop on, 2013, pp. 30-35. 
[5] Vimala G. and Mohideen S. K., "Automatic detection of optic disk and exudate from retinal images using clustering algorithm," in Intelligent Systems and Control (ISCO), 2013 7th International Conference on, 2013, pp. 280-284.

[6] Sreng S., Takada J.-I., Maneerat N., Isarakorn D., Pasaya B., Panjaphongse R., et al., "Automatic exudate extraction for early detection of Diabetic Retinopathy," in Information Technology and Electrical Engineering (ICITEE), 2013 International Conference on, 2013, pp. 31-35.

[7] Ramasubramanian B. and Mahendran G., "An efficient integrated approach for the detection of exudates and Diabetic Maculopathy in colour fundus images," Advanced Computing, vol. 3, 2012.

[8] Sopharak A., Uyyanonvara B., Barman S., and Williamson T. H., "Automatic detection of diabetic retinopathy exudates from non-dilated retinal images using mathematical morphology methods," Computerized Medical Imaging and Graphics, vol. 32, pp. $720-727,2008$.

[9] Karegowda A. G., Nasiha A., Jayaram M., and Manjunath A., "Exudates detection in retinal images using back propagation neural network," International Journal of Computer Application, vol. 25, pp. 25-31, 2011.

[10] Eadgahi M. G. F. and Pourreza H., "Localization of hard exudates in retinal fundus image by mathematical morphology operations," in Computer and Knowledge Engineering (ICCKE), 2012 2nd International eConference on, 2012, pp. 185-189.

[11] Tripathi S., Singh K. K., Singh B., and Mehrotra A., "Automatic detection of exudates in retinal fundus Images using differential morphological profile," International Journal of Engineering \& Technology (0975-4024), vol. 5, 2013.

[12] El Abbadi N. K. and Al-Saadi E. H., "Automatic detection of exudates in retinal images," International Journal of Computer Science Issues (IJCSI), vol. 10, 2013.
[13] Vandarkuzhali T., Ravichandran C., and Preethi D., "Detection of exudates caused by Diabetic Retinopathy in fundus retinal image using fuzzy K Means and Neural Network."

[14] Franklin S. W. and Rajan S. E., "Diagnosis of diabetic retinopathy by employing image processing technique to detect exudates in retinal images," Image Processing, IET, vol. 8, pp. 601-609, 2014.

[15] Zhang X., Thibault G., Decencière E., Marcotegui B., Laÿ B., Danno R., et al., "Exudate detection in color retinal images for mass screening of diabetic retinopathy," Medical image analysis, vol. 18, pp. 10261043,2014

[16] Gonzalez R. C., Woods R. E., and Eddins S. L., 2004, Digital image processing using MATLAB, Upper Saddle River, N. J: Pearson Prentice Hall, 2.

[17] Setiawan A. W., Mengko T. R., Santoso O. S., and Suksmono A. B., "Color retinal image enhancement using clahe," in ICT for Smart Society (ICISS), 2013 International Conference on, 2013, pp. 1-3.

[18] Shi G., Gao B., and Zhang L., "The optimized k-means algorithms for improving randomly-initialed midpoints," in Measurement, Information and Control (ICMIC), 2013 International Conference on, 2013, pp. 1212-1216.

[19] Hashim F., Salem N., and Seddik A., "Optic disc boundary detection from digital fundus images," Journal of Medical Imaging and Health Informatics, vol. 5, pp. 50-56, 2015.

[20] Mendonça A. M., Sousa A., Mendonça L., and Campilho A., "Automatic localization of the optic disc by combining vascular and intensity information," Computerized Medical Imaging and Graphics, vol. 37, pp. 409-417, 2013.

[21] Hoover A., "STARE Database," Avilable: http://www.ces.clemson.edu/ ahoover/stare/

[22] Amel F., Mohammed M., and Abdelhafid B., "Improvement of the hard exudates detection method used for computer-aided diagnosis of diabetic retinopathy," International Journal of Image, Graphics and Signal Processing (IJIGSP), vol. 4, p. 19, 2012 